\title{
An Encounter-Type Multi-Fingered Master Hand Using Circuitous Joints
}

\author{
Shuhei Nakagawara, Hiroyuki Kajimoto, \\ Naoki Kawakami, and Susumu Tachi \\ Graduate School of Information Science and Technology \\ The University of Tokyo \\ 7-3-1 Hongo, Bunkyo-ku, Tokyo, Japan \\ \{nkgwr,kaji,kawakami,tachi\}@star.t.u-tokyo.ac.jp
}

\author{
Ichiro Kawabuchi \\ TechExperts Incorporation \\ 4-15-5 Omori Nishi, Ota-ku, Tokyo, Japan \\ ichiro_kawabuchi@post.pioneer.co.jp
}

\begin{abstract}
We have developed a new type of master hand to control a dexterous slave robot hand for telexistence. Our master hand has two features. One is a compact exoskeleton mechanism called "circuitous joint," which covers wide workspace of an operator's finger. Another is the encountertype force feedback that enables unconstrained motion of the operator's finger and natural contact sensation. In this paper, the mechanism and control method of our master hand are introduced and experimental master-slave finger control is conducted.
\end{abstract}

Index Terms - Master hand, Encounter-type haptic device, Force feedback, Telexistence

\section{INTRODUCTION}

Recently some dexterous robot hands have been developed. To control these hands (slave hands), an operator must wear master hands. The master hands can be divided into two categories. One is exoskeleton type such as CyberGrasp [1]. However, most conventional exoskeleton type master hands tend to be bulky and require an operator wearing a glove to measure the state of an operator's finger. The other is endoskeleton type such as Rutgers Master II [2]. Although it is light-weight and compact and does not need a glove, it does not allow complete fist closure, due to the placement of the actuators in the palm.

We have developed a new type of master hand (Fig. 1) to improve these disadvantages. Our master hand has two features as follows.

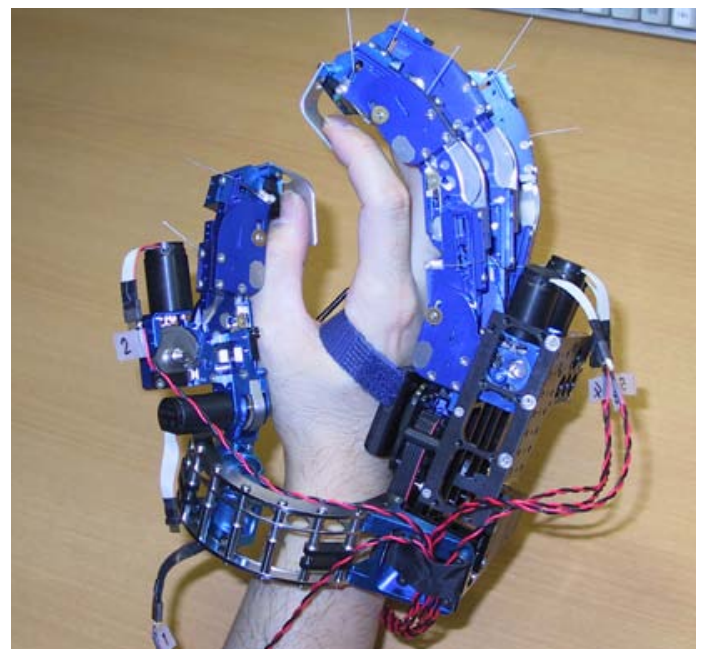

Fig. 1 Appearance of the master hand
One is the compact exoskeleton mechanism of the master hand's finger contrived to cover wide workspace of an operator. Possible ways to place an exoskeleton mechanism are to place it over (parallel joint) or beside (coaxial joint) an operator's finger. To place it over the operator's finger, there is a problem that master finger disturbs the motion of the operator's finger when the finger is bent as shown in Fig. 2. However, to place it beside an operator's finger is difficult, because there is little space to place it. To solve this problem, we propose "circuitous joint" that coincides joint axis of the master hand with that of the operator by extending the link length in proportion to the joint angular displacement (Fig. 3).

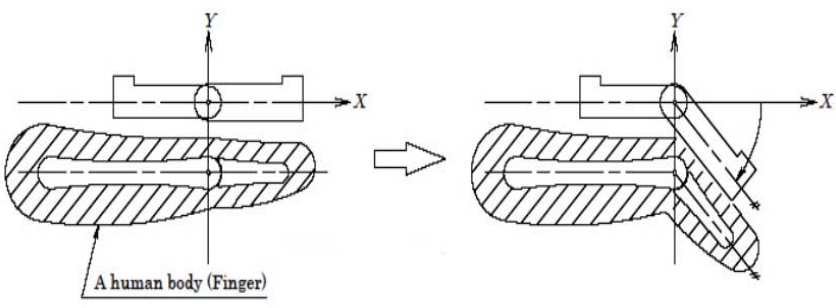

Fig. 2 Parallel joint disturbing operator's motion when finger is bent

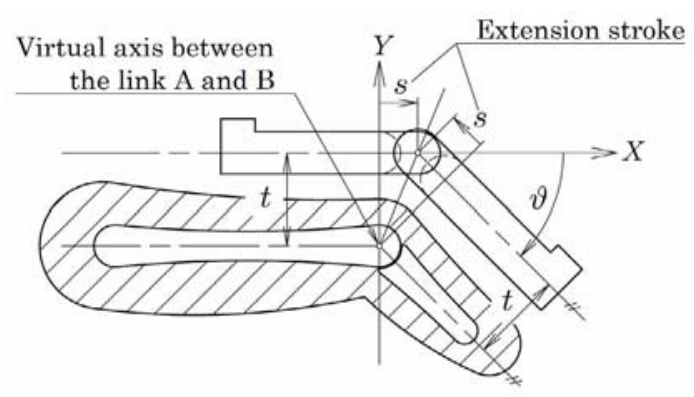

Fig. 3 Basic scheme of the circuitous joint

The other is the encounter-type force feedback. Encounter-type haptic device was first proposed by McNeely [3], and Tachi [4] proposed a similar idea independently. Encounter-type device stays at the location of the object in the remote world and waits for an operator to encounter it. As shown in Fig. 4, our encounter-type master hand's finger usually follows the operator's finger without contact. It enables him to touch nothing when the slave hand contacts nothing. When slave hand touches an object, master finger contacts the operator's finger and provides resistive force. Therefore, our master hand is able to provide both perfect unconstrained motion and natural touch sensation. 
In this paper, we introduce our encounter-type force feedback multi-fingered master hand. Section II details the mechanism including "circuitous joint" of the master hand. Section III describes encounter-type force feedback and the control of the master hand. Experimental master-slave control using our master hand is given in section IV.

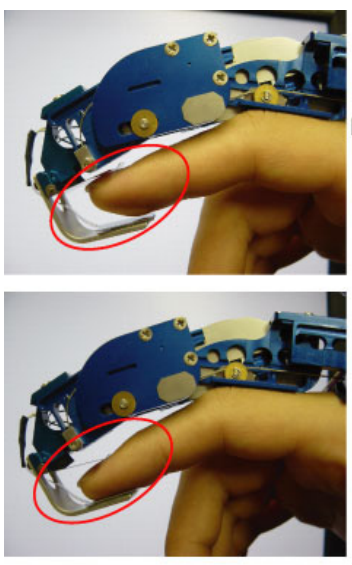

Master hand

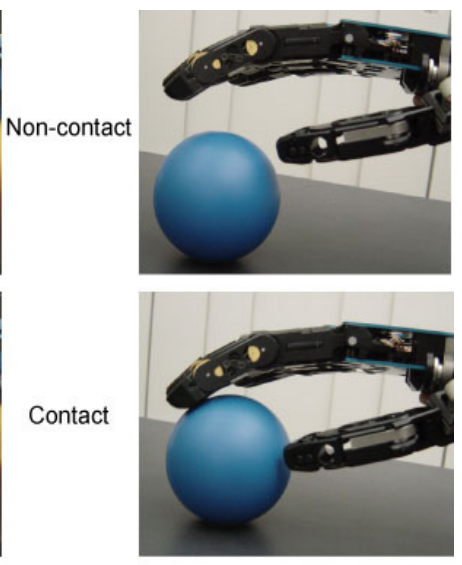

Slave hand
Fig. 4 Concept of the encountered-type force feedback master hand

\section{MEChANISM OF THE MASTER HAND}

\section{A. Circuitous Joint}

The principle of the circuitous joint is shown in Fig. 5. In order to extend the length of the link in proportion to joint angular displacement, the mechanism that consists of rack and sector gear is adopted from the viewpoint of the simplicity of structure, the certainty of movement, and high rigidity. A sector gear rotates on a rack by relative rotation of two links, and movement of the gear center axis produces flexible movement of the link with the rack. Since two links have to make extension stroke together, two sets of a rack and a sector gear are used in the reverse direction.

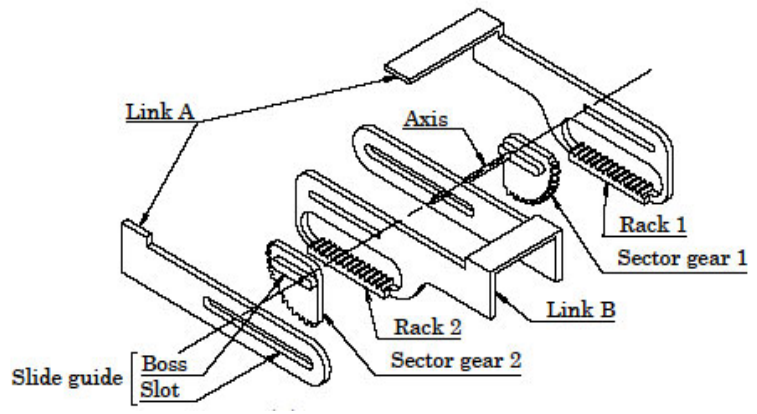

Fig. 5 Disassembled view of the circuitous joint

To coincide the rotation axis of the master finger and that of the operator in all rotation angles, the relation between the rotation angular displacement and the extension stroke of the link must be nonlinear. This means that the rectilinear rack and the sector gear whose radius is constant are not appropriate. However, it is possible to keep the error to acceptable range. We obtained the radius that minimizes the maximum value of the error between ideal position and real one of fingertip position. Meanwhile, we contrived to avoid the friction of the master finger and an operator. As shown in Fig. 6, we placed the rack on the upper side of the sector gear to enlarge the distance between the master hand mechanism and an operator. In order to keep the reverse gears in line with the rack and the sector gear, sliders are used (Fig. 7).

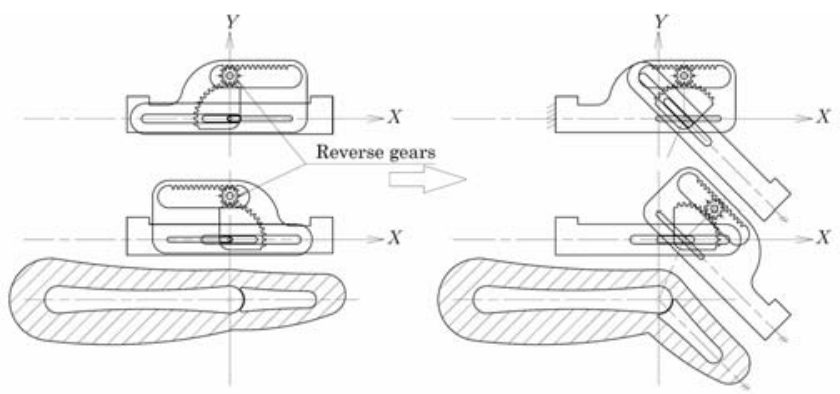

Fig. 6 The circuitous joint with upper side racks and reverse gears

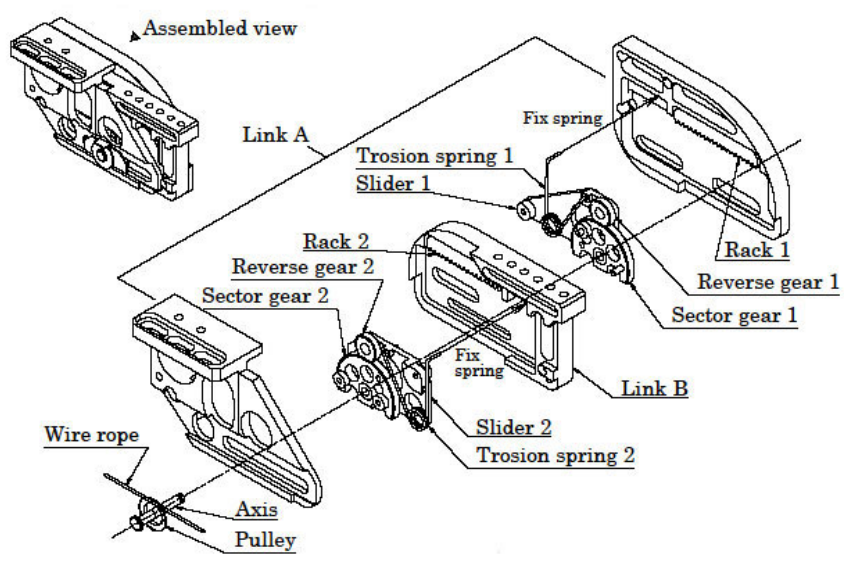

Fig. 7 The circuitous joint mechanism with sliders

Our master finger consists of three circuitous joints in series corresponding to human finger's three joints (DIP, PIP, MCP joint). The length of the finger is different from each other, so the length of the link is adjustable by changing the position of connecting screws. Additionally, in order to make the width short, a part of each link overlaps alternately (Fig. 8). The width of the master finger is about $19[\mathrm{~mm}]$ which is the same as human. As shown in Fig. 9, the length of the master finger in flexion becomes up to about twice as long as it in extension.

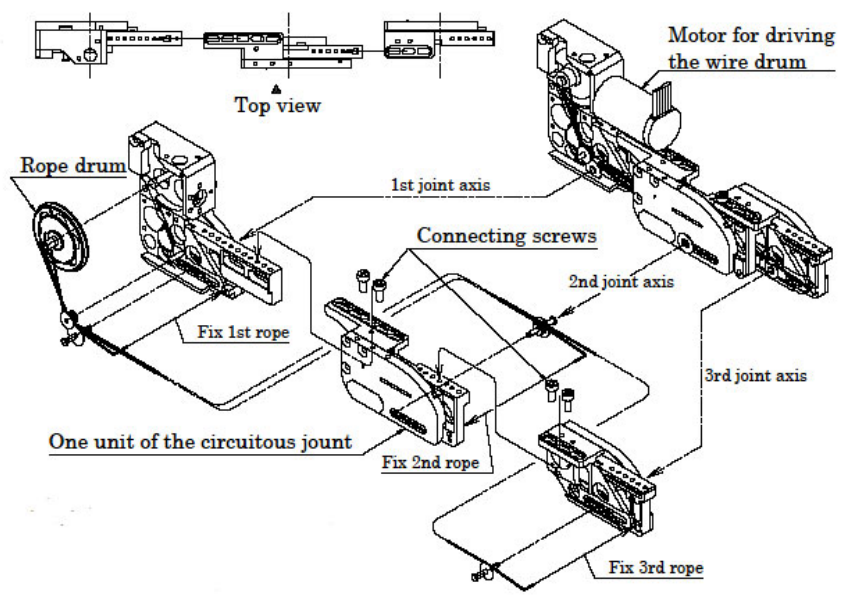

Fig. 8 Adjustable serial connection of three joint units 


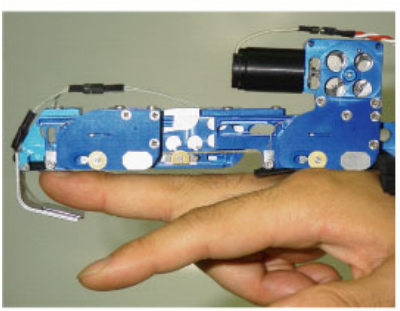

extension

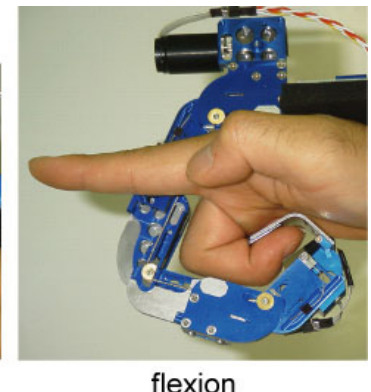

flexion
Fig. 9 Bending motion of the practical mechanism with three joints

\section{B. Driving Method}

Driving motors must not be near the joints because mechanism gets larger and heavier. Therefore, we used wire mechanism as shown in Fig. 10. A wire is set between two links via a pulley on the sector gear's center axis, and a motor pulls the wire to the extensional direction. Since the wire cannot produce a force in an opposite direction, a compression spring is used to produce a force in the direction of flexion (Fig. 11).

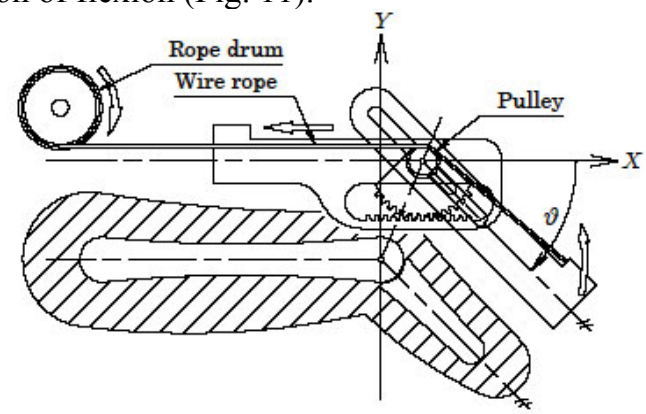

Fig. 10 Driving method by pulling a wire rope

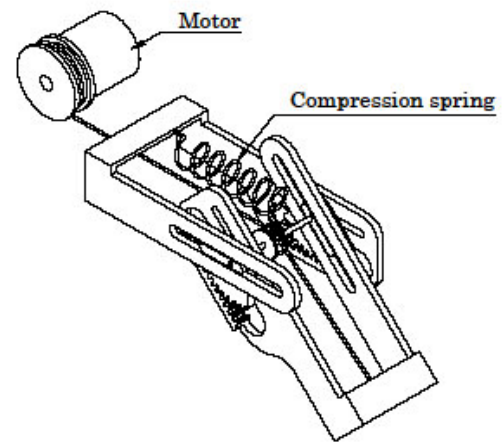

Fig. 11 Built-in compression spring

As each joint is driven by one rope, three wires are used for one finger which are driven by one motor (FaulHaber GmbH\&Co., 1724SR). Three circuitous joints are coupled and each rate of rotation is decided by the radius of the drum (Fig. 12). The maximum resistive force applied is about $8[\mathrm{~N}]$. The approximate maximum velocity and acceleration of master fingertip are shown in Table I. From a preliminary experiment the maximum velocity and acceleration of human fingertip are appropriately $1.0[\mathrm{~m} / \mathrm{s}]$ and $300\left[\mathrm{~m} / \mathrm{s}^{2}\right]$ respectively. Our master hand has enough ability to follow operator's normal motion without contact.
TABLE I

Approximate maximum velocity and acceleration of the master finger

\begin{tabular}{|c|c|c|c|}
\hline \multicolumn{2}{|c|}{} & Extension & Flexion \\
\hline \multirow{2}{*}{$\begin{array}{c}\text { Maximum joint angular velocity } \\
{[\mathrm{rad} / \mathrm{s}]}\end{array}$} & DIP & 4.5 & 5.0 \\
\cline { 2 - 4 } & PIP & 6.8 & 7.5 \\
\cline { 2 - 4 } & MCP & 4.5 & 5.0 \\
\hline \multirow{2}{*}{$\begin{array}{c}\text { Maximum joint angular acceleration } \\
{\left[\mathrm{rad} / \mathrm{s}^{2}\right]}\end{array}$} & DIP & 1500 & 1500 \\
\cline { 2 - 4 } & PIP & 2300 & 2300 \\
\cline { 2 - 4 } & MCP & 1500 & 1500 \\
\hline Maximum fingertip velocity [m/s] & & 0.85 & 0.95 \\
\hline Maximum fingertip acceleration $\left[\mathrm{m} / \mathrm{s}^{2}\right]$ & 300 & 350 \\
\hline
\end{tabular}

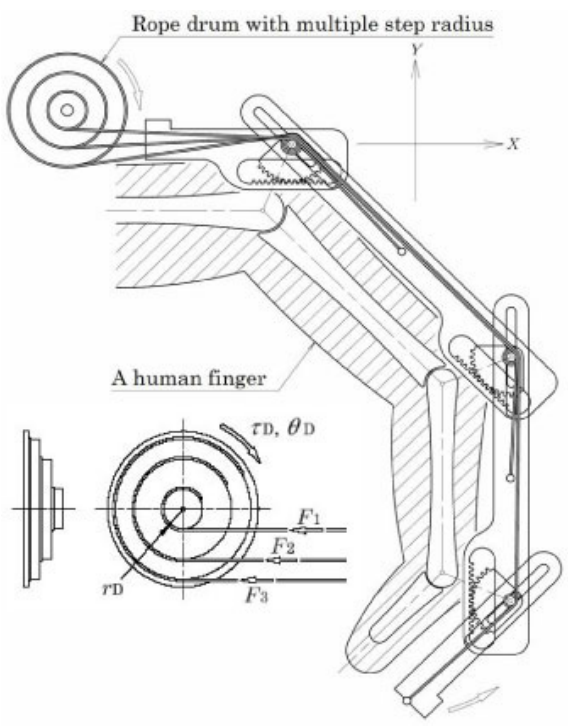

Fig. 12 Coupled driving method of the master finger with pulling all ropes by one drum

C. Sensing for Encounter-Type Force Feedback

For encounter-type force feedback, the distance between tip of the master finger and that of the operator and the force between them must be measured. As shown in Fig. 13, we set a photo reflector (Sanyo Electric Co., Ltd., SPI315-34), a reflecting plate (thin stainless plate with hinge and spring), and a thin force sensor (Nitta Corporation, FlexiForce) at the tip of the master finger.

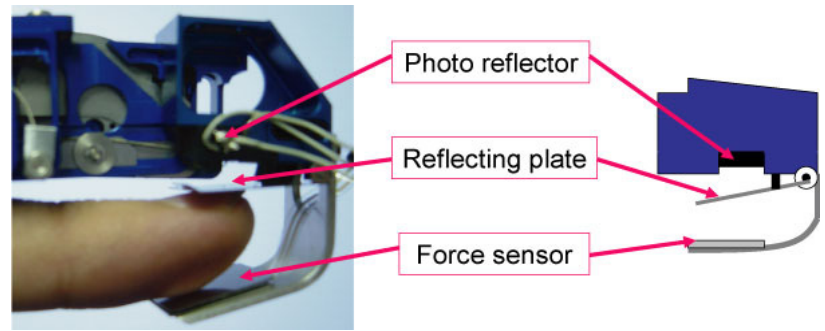

Fig. 13 Sensors put on the tip of master finger

\section{CONTROL OF THE MASTER HAND}

\section{A. The Model to Calculate the Torque}

First, we derived the relation between rope tension and joint torque of a circuitous joint. Parameters were defined as shown in Fig. 14. 


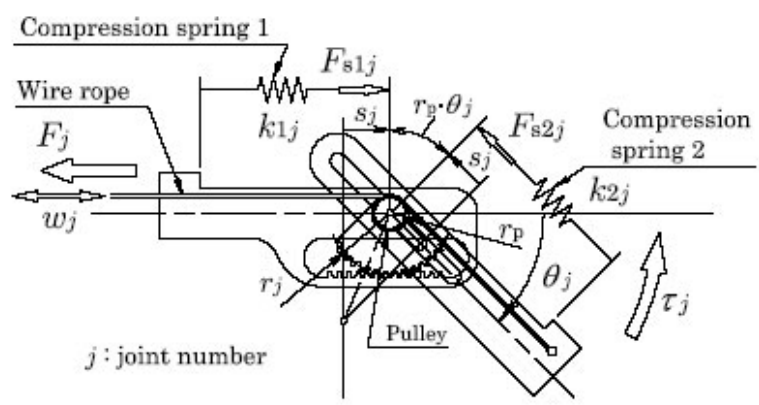

$(j:$ Joint number

$\theta_{j}:$ Joint angular displacement

$s_{j}:$ Joint stretching displacement

$r_{j}$ : Radius of pitch circle of the sector gear

$r_{p}:$ Radius of the rope pulley

$k_{1 j}$ : Spring constant of the compression spring 1

$k_{2 j}$ :Spring constant of the compression spring 2

$\tau_{j}:$ Joint torque

$F_{j}:$ Rope tension

$w_{j}:$ Rope stretch

$F_{s 1 j}$ : Spring force of the compression spring 1

$F_{s 2 j}$ : Spring force of the compression spring 1

$F_{s 1 j}{ }^{\prime}$ : Spring force of the compression spring 1 when $\theta_{j}=0$

$F_{s}{ }^{\prime}{ }_{2 \mathrm{j}}$ : Spring force of the compression spring 2 when $\theta_{j}=0$ )

Fig. 14 Relation between rope tension and joint torque at a circuitous joint

The relation between rope stretch and joint angular displacement is

$$
\begin{aligned}
s_{j} & =r_{j} \cdot \theta_{j}, \\
w_{j} & =2 s_{j}+r_{p} \cdot \theta_{j} .
\end{aligned}
$$

When motor angular displacement changes from $\theta_{j}$ to 0 , the work done by rope stretch is $F_{j} \cdot w_{j}$, the work done by joint torque is $\tau_{j} \cdot \theta_{j}$, and the energy displacement of compression spring 1,2 are $F_{s}{ }^{\prime}{ }_{1 j} \cdot s_{j}-k_{1 j} \cdot s_{j}{ }^{2} / 2$ and $F_{s}{ }^{\prime}{ }_{2 j} \cdot s_{j}-k_{2 j} \cdot s_{j}{ }^{2} / 2$. Therefore, the relation between rope tension and joint torque is derived by the law of conservation of energy as follows,

$$
F_{j} \cdot w_{j}=\tau_{j} \cdot \theta_{j}+\left(F_{s 1 j}^{\prime} \cdot s_{j}-k_{1 j} \cdot s_{j}{ }^{2} / 2\right)+\left(F_{s}{ }^{\prime}{ }_{2 j} \cdot s_{j}-k_{2 j} \cdot s_{j}{ }^{2} / 2\right) \text {. }
$$

From (1), (2) and (3), rope tension is

$$
\begin{gathered}
w_{j}=\left(2 r_{j}+r_{p}\right) \theta_{j}, \quad \text { (4) } \\
F_{j}=\frac{\tau_{j}}{2 r_{j}+r_{p}}+\frac{\left(F_{s}{ }^{\prime}{ }_{1 j}+F_{s}{ }^{\prime}{ }_{2 j}\right) r_{j}}{2 r_{j}+r_{p}}+\frac{\left(k_{1 j}+k_{2 j}\right) r_{j}{ }^{2}}{2\left(2 r_{j}+r_{p}\right)} \theta_{j} .
\end{gathered}
$$

The second and third term in the right-hand side of (5) is the effect of the compression spring, so if we treat it with disregard, (5) is written as

$$
F_{j}=\tau_{j} /\left(2 r_{j}+r_{p}\right) .
$$

Equation (6) indicates that the relation between rope tension and joint torque is linear, and joint angular displacement has no effect. That is, the driving method using wire rope has an advantage that the control of joint torque is easy. We omit the effect of the compression spring hereafter for simple expression of equation.
When some circuitous joints are connected in series like our master hand, the sum of all rope tensions works as the joint torque. Thus, the rope tension of the root joint that has to generate a great force is divided into some ropes. In the case of our master hand, as shown in Fig. 15, three ropes are used. The relation between joint torque and three ropes tension at the jth joint is

$$
\tau_{j}=\sum_{m=j}^{3} F_{m}\left(2 r_{j}+r_{p}\right) \quad(j=1,2,3) .
$$

Next, given the joint angular displacements $\theta_{1}, \theta_{2}, \theta_{3}$ and contact force $F_{T}$ acted on $\mathrm{T}$ (at the tip of the finger), the rope tension is derived as follows. The relation between jth joint torque and contact force is expressed as

$$
\tau_{j}=\overrightarrow{J_{j} T} \times \overrightarrow{F_{T}}(j=1,2,3) .
$$

where $\overrightarrow{J_{j} T}$ is a vector from joint $J_{i}$ to $\mathrm{T}$.

By substituting (8) to (7), (7) is

$\left\{\begin{array}{l}\left(F_{1}+F_{2}+F_{3}\right)\left(2 r_{1}+r_{p}\right)=\overrightarrow{J_{1} T} \times \overrightarrow{F_{T}}, \\ \left(F_{2}+F_{3}\right)\left(2 r_{2}+r_{p}\right)=\overrightarrow{J_{2} T} \times \overrightarrow{F_{T}}, \\ F_{3}\left(2 r_{3}+r_{p}\right)=\overrightarrow{J_{3} T} \times \overrightarrow{F_{T}} .\end{array}\right.$

From (9), rope extension is

$\left\{\begin{array}{l}F_{1}=\frac{\overrightarrow{J_{1} T} \times \overrightarrow{F_{T}}}{2 r_{1}+r_{p}}-\frac{\overrightarrow{J_{2} T} \times \overrightarrow{F_{T}}}{2 r_{2}+r_{p}}-\frac{\overrightarrow{J_{3} T} \times \overrightarrow{F_{T}}}{2 r_{3}+r_{p}}, \\ F_{2}=\frac{\overrightarrow{J_{2} T} \times \overrightarrow{F_{T}}}{2 r_{2}+r_{p}}-\frac{\overrightarrow{J_{2} T} \times \overrightarrow{F_{T}}}{2 r_{2}+r_{p}}, \\ F_{3}=\frac{\overrightarrow{J_{3} T} \times \frac{\overrightarrow{F_{T}}}{2 r_{3}+r_{p}} .}{} .\end{array}\right.$

Additionally, from (4), rope stretch is

$\left\{\begin{array}{l}w_{1}=\left(2 r_{1}+r_{p}\right) \theta_{1}, \\ w_{2}=\left(2 r_{1}+r_{p}\right) \theta_{1}+\left(2 r_{2}+r_{p}\right) \theta_{2}, \\ w_{3}=\left(2 r_{1}+r_{p}\right) \theta_{1}+\left(2 r_{2}+r_{p}\right) \theta_{2}+\left(2 r_{3}+r_{p}\right) \theta_{3} .\end{array}\right.$

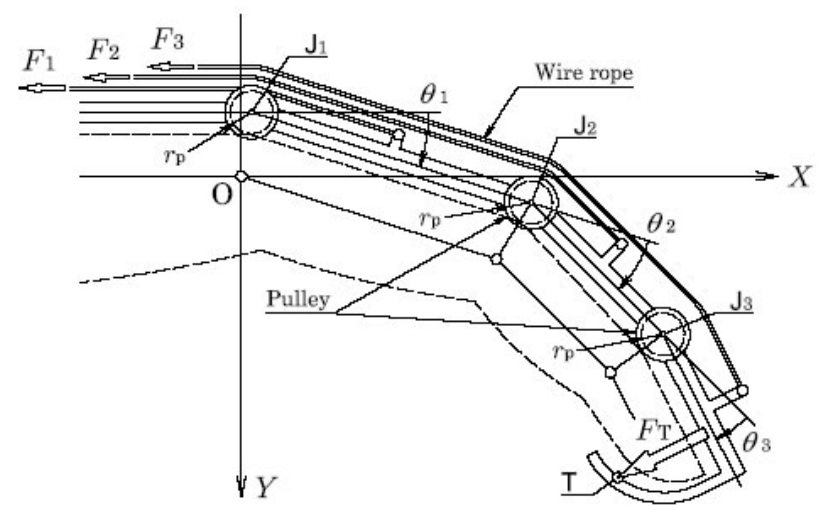

Fig. 15 Force equilibrium on the master finger mechanism

As mentioned above, our master finger is driven with three coupled joints whose ratio (constant) is

$$
\theta_{1}: \theta_{2}: \theta_{3}=1: u: v \text {. }
$$

We assume the following three conditions; (i) no friction of master hand mechanism, (ii) contact force acted on $\mathrm{T}$ intersects perpendicularly with the direction of the link length, (iii) point of application of the contact force is considered to be "Virtual T" in Fig. 16.

Then from (12), (11) is 
$\left\{\begin{array}{l}w_{1}=\left(2 r_{1}+r_{p}\right) \theta_{1}, \\ w_{2}=\left(\left(2 r_{1}+r_{p}\right)+\left(2 r_{2}+r_{p}\right) u\right) \theta_{1}, \\ w_{3}=\left(\left(2 r_{1}+r_{p}\right)+\left(2 r_{2}+r_{p}\right)+\left(2 r_{3}+r_{p}\right) v\right) \theta_{1} .\end{array}\right.$

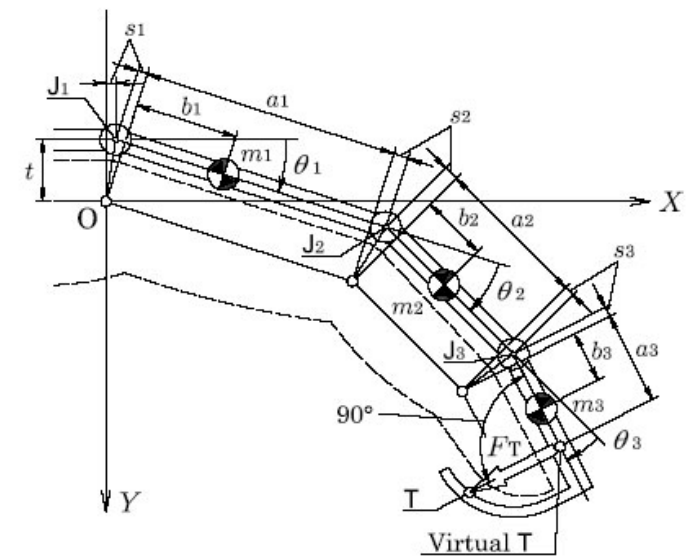

$\left(\begin{array}{l}t: \text { Offset between the master finger and an operator's finger } \\ a_{j}: \text { Length of the link } \\ b_{j}: \text { Center of mass of the link } \\ m_{j}: \text { weight of the link }\end{array}\right)$

Fig. 16 Specifications of the master finger mechanism

Since the radius of each drum is proportional to the rope stretch given in (13), torque to rotate the drum is

$$
\begin{aligned}
& \tau_{D}=\left(F_{1}+\frac{\left(2 r_{1}+r_{p}\right)+\left(2 r_{2}+r_{p}\right) u}{2 r_{1}+r_{p}} F_{2}+\frac{\left(2 r_{1}+r_{p}\right)+\left(2 r_{2}+r_{p}\right) u+\left(2 r_{3}+r_{p}\right) v}{2 r_{1}+r_{p}} F_{3}\right) r_{D} \\
& \left(\begin{array}{l}
r_{D}: \text { Radius of the drum which reels the shotest rope } \\
\tau_{D}: \text { Torque to rotate the drum } \\
\theta_{D}: \text { Rotation angular displacement of the drum }
\end{array}\right) .
\end{aligned}
$$

Additionally, each vector is

$$
\left\{\begin{aligned}
\overrightarrow{F_{T}}= & F_{T}\left(\begin{array}{c}
-\sin \left(\theta_{1}+u \theta_{1}+v \theta_{1}\right) \\
\left.\cos \theta_{1}+u \theta_{1}+v \theta_{1}\right)
\end{array}\right), \\
\overrightarrow{J_{1} T}= & \left(a_{1}+r_{1} \cdot \theta_{1}+r_{2} \cdot u \theta_{1}\right)\left(\begin{array}{c}
\cos \theta_{1} \\
\sin \theta_{1}
\end{array}\right)+\left(a_{2}+r_{2} \cdot u \theta_{1}+r_{3} \cdot v \theta_{1}\right)\left(\begin{array}{l}
\left.\cos \theta_{1}+u \theta_{1}\right) \\
\sin \left(\theta_{1}+u \theta_{1}\right)
\end{array}\right) \\
& +\left(a_{3}+r_{3} \cdot v \theta_{1}\right)\left(\begin{array}{c}
\left.\cos \theta_{1}+u \theta_{1}+v \theta_{1}\right) \\
\sin \left(\theta_{1}+u \theta_{1}+v \theta_{1}\right)
\end{array}\right) \\
\overrightarrow{J_{2} T}= & \left(a_{2}+r_{2} \cdot u \theta_{1}+r_{3} \cdot v \theta_{1}\right)\left(\begin{array}{c}
\left.\cos \theta_{1}+u \theta_{1}\right) \\
\sin \left(\theta_{1}+u \theta_{1}\right)
\end{array}\right)+\left(a_{3}+r_{3} \cdot v \theta_{1}\right)\left(\begin{array}{l}
\left.\cos \theta_{1}+u \theta_{1}+v \theta_{1}\right) \\
\sin \left(\theta_{1}+u \theta_{1}+v \theta_{1}\right)
\end{array}\right) \\
\overrightarrow{J_{3} T}= & \left(a_{3}+r_{3} \cdot v \theta_{1}\right)\left(\begin{array}{c}
\left.\cos \theta_{1}+u \theta_{1}+v \theta_{1}\right) \\
\sin \left(\theta_{1}+u \theta_{1}+v \theta_{1}\right)
\end{array}\right)
\end{aligned}\right),
$$

In (15), $\theta_{1}$ is decided from (13), motor angular displacement $\theta_{m}$ and reduction gear ratio $r_{g}$ as

$$
\begin{aligned}
\theta_{1} & =\frac{w_{3}}{\left(2 r_{1}+r_{P}\right)+\left(2 r_{2}+r_{p}\right) u+\left(2 r_{3}+r_{P}\right) v} \\
& =\frac{r_{D} r_{g} \theta_{m}}{\left(2 r_{1}+r_{P}\right)+\left(2 r_{2}+r_{p}\right) u+\left(2 r_{3}+r_{P}\right) v} .
\end{aligned}
$$

Finally, by substituting (10) and (15) to (14), the relation between joint angular displacement $\theta_{1}$, fingertip load $F_{T}$, and torque $\tau_{D}$ is obtained.

\section{B. Control Method}

As shown in Fig.14, for encounter-type force feedback, the master finger is controlled to keep the distance between the operator's fingertip and its tip when the slave hand does not contact an object, which is a simple position control. When slave hand touches an object, master finger is controlled to produce a force that is equal to the force applied on the slave finger (force control). At the same time, the slave finger is always controlled to take the same position as that of the master finger (position control). This bilateral control is so-called force-reflecting servo type. In Fig. $17, X$ is position, $F$ is force, $d$ is the distance between tip of the master finger and that of the operator, measured by an optical sensor, and $d_{d}$ is its desired value. Each suffix m, s represents master and slave respectively.

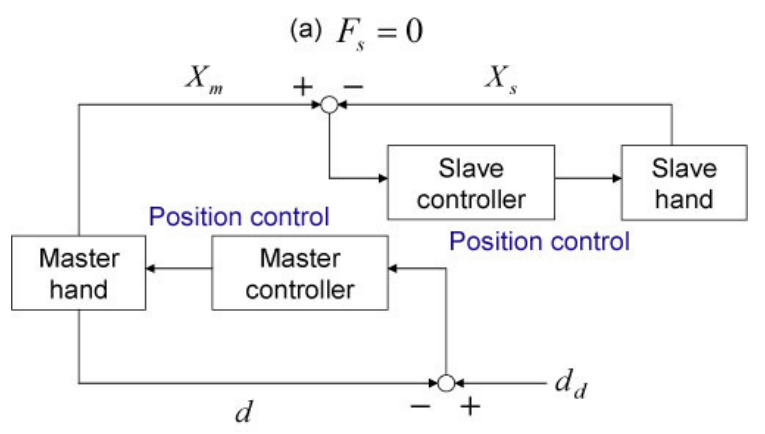

(b) $F_{s}>0$

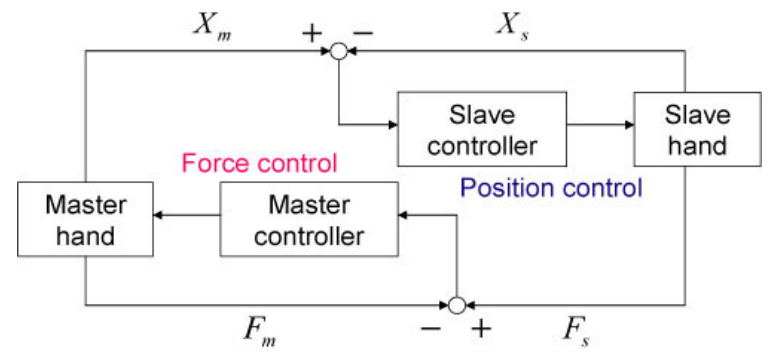

Fig. 17 Block diagram of control

\section{EXPERIMENT}

\section{A. Confirmation of the Model of the Master Hand}

To confirm the control model of the master hand mentioned in III-A, a control experiment is conducted. In III-A, we disregarded the effect of compression springs and mass of the links above, but in fact we cannot ignore it. When we regard the mass of the link, (8) is

$$
\tau_{j}=\overrightarrow{J_{j} T} \times \overrightarrow{F_{T}}+\sum_{m=j}^{3}\left(\overrightarrow{J_{j} m_{m}} \times m_{m} \vec{G}\right)(j=1,2,3) .
$$

$\vec{G}$ is the gravity direction. Considering the effect of compression springs and mass of links, using (5), (13), and (17), the relation between motor torque and resistive force is written as

$$
\begin{aligned}
& \tau_{D}=\frac{r_{D}}{2 r_{1}+r_{p}}\left[\left(\overrightarrow{J_{1} T} \times \overrightarrow{F_{T}}+\overrightarrow{J_{1} m_{1}} \times m_{1} \vec{G}+\overrightarrow{J_{1} m_{2}} \times m_{2} \vec{G}+\overrightarrow{J_{1} m_{3}} \times m_{3} \vec{G}\right.\right. \\
& \left.+\left(F_{s 11^{\prime}}+F_{s 21}{ }^{\prime}\right) r_{1}-\frac{1}{2}\left(k_{11}+k_{21}\right) r_{1}^{2} \theta_{1}\right\} \\
& +u\left\{\overrightarrow{J_{2} T} \times \overrightarrow{F_{T}}+\overrightarrow{J_{2} m_{2}} \times m_{2} \vec{G}+\overrightarrow{J_{2} m_{3}} \times m_{3} \vec{G}+\left(F_{s 12}{ }^{\prime}+F_{s 22}{ }^{\prime}\right) r_{2}-\frac{1}{2}\left(k_{12}+k_{22}\right) r_{2}^{2} \theta_{2}\right\} \\
& \left.+v\left\{\overrightarrow{J_{3} T} \times \overrightarrow{F_{T}}+\overrightarrow{J_{3} m_{3}} \times m_{3} \vec{G}+\left(F_{s 13}^{\prime}+F_{s 23}{ }^{\prime}\right) r_{3}-\frac{1}{2}\left(k_{13}+k_{23}\right) r_{3}^{2} \theta_{3}\right\}\right] .
\end{aligned}
$$


We compared motor torque generated by PID control and theoretical one calculated by (18). The theoretical motor torque is calculated by the following parameters,

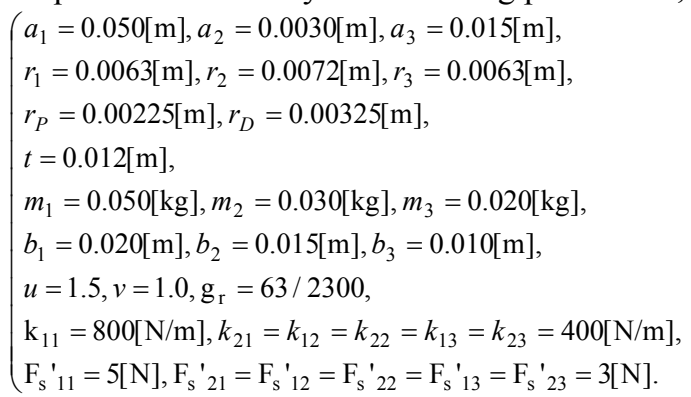

The result is shown in Fig. 18. The vertical axis is motor angular displacement and the horizontal axis is the ratio of motor torque to contact force between the operator and master finger. An obvious accordance of real and theoretical value can be seen.

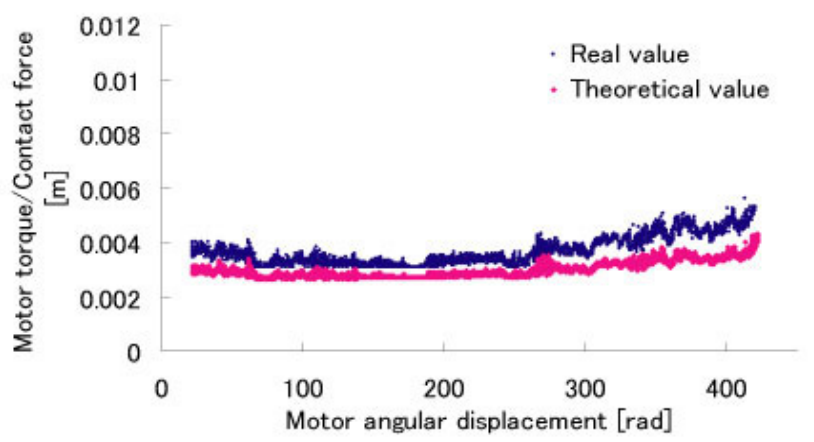

Fig. 18 Relation between real value and theoretical one

\section{B. Experimental Master-Slave Control}

Using our master hand and a slave hand [5], experimental master-slave control is conducted. The extension and flexion motion of three joints of this slave hand's finger is coupled like our master hand. Therefore, we only have to control the motor's rotation angle of the slave finger in accordance with that of the master finger. We used only one finger because four fingers except thumb are controlled in the same way. In the first period $(0 \sim 5$ seconds), slave finger is not in contact with an object, and in the latter half (5 13 seconds), slave finger touches the object.

Results are shown in Fig. 19. As shown in Fig. 19 (top), the slave finger can follow the motion of the master finger. As shown in Fig. 19 (middle), when the slave finger doesn't contact an object, the master finger mechanism can keep a distance from an operator's finger. As shown in Fig. 19 (bottom), when the slave finger contacts the object, master finger can produce as much resistive force as the slave finger's.

\section{CONCLUSION AND FUTURE WORK}

In this paper, we introduced an encounter-type multifingered master hand focusing on its two features. One is its compact exoskeleton mechanism called circuitous joint, which can cover wide workspace of the operator. The other is the encounter-type force feedback which enables an operator to feel real free and real touch sensations. We derived a torque calculation model of the master hand and confirmed its accuracy by an experiment. Additionally, we examined the precision of the encounter-type force feedback by experimental master-slave control.

Index finger, middle finger, fourth finger, and fifth finger can be controlled by the method mentioned above. However, thumb of human hand has very complicated structure and our next step is to realize the non-contact motion following of thumb.

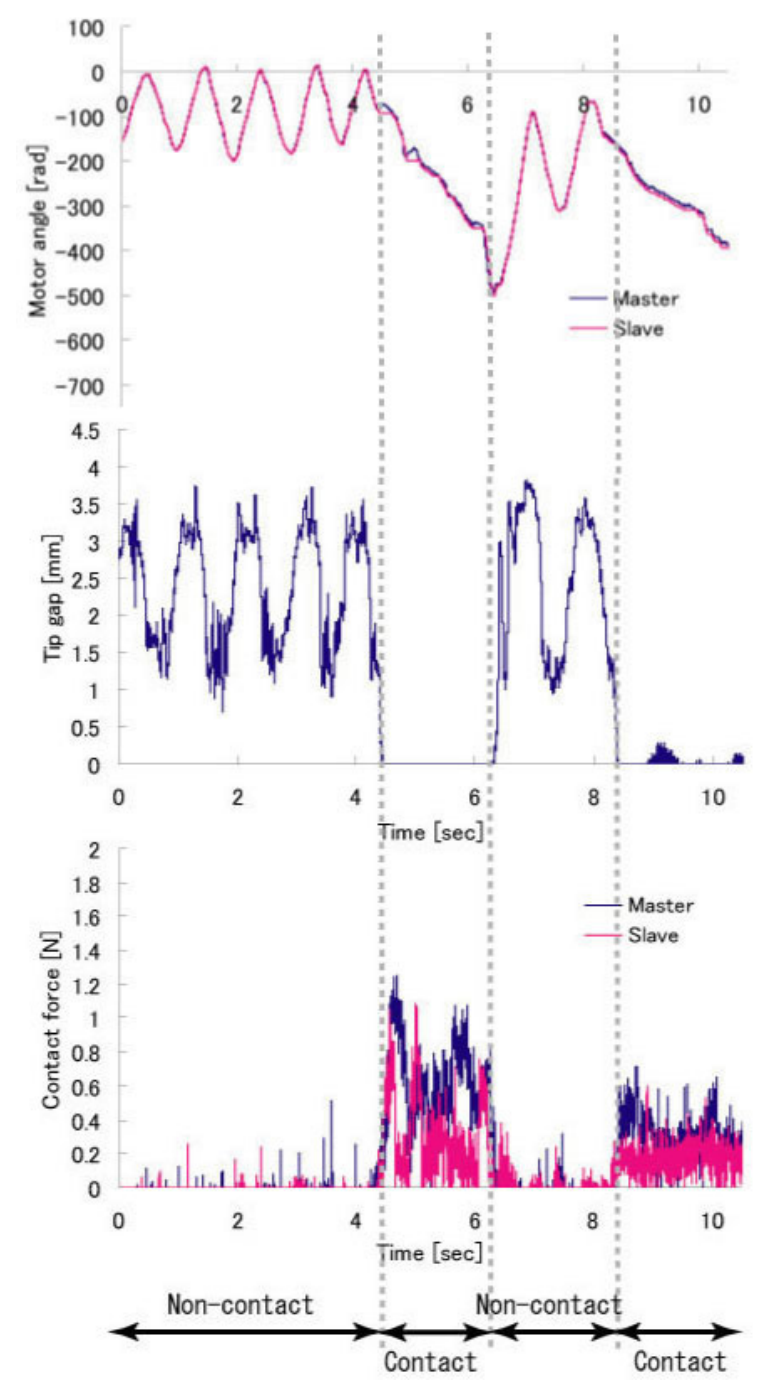

Fig. 19 Motor's rotation angle (top), distance between operator's and master's fingertip (middle), and contact force (bottom) of the master and slave.

\section{REFERENCES}

[1] Immersion Corporation, "http://www.immersion.com/3d/products/cyber_grasp.php."

[2] M.Bouzit, G.Burdea, G.Popescu, R. Boian, "The Rutgers Master II New Design Force-Feedback Glove," Proc. of IEEE/AMSE Trans. on Mechatronics, Vol. 7, No. 2, pp. 256-263, 2002.

[3] W.A.McNeely: "Robotic Graphics, "A New Approach to Force Feedback for Virtual Reality," Proc. of 1993 IEEE Virtual Reality Annual International Symposium (VRAIS '93), pp. 336-341, 1993.

[4] S.Tachi, T.Maeda, R.Hirata, and H.Hoshino, "A Construction Method of Virtual Haptic Space," Proc. of the 4th International Conference on Artificial Reality and Telexistence (ICAT '94), pp. 131-138, 1994.

[5] K.Hoshino and I.Kawabuchi: "A humanoid robotic hand performing the sign language motions," Proc. of 2003 International Symposium on Micromechatronics and Human Science (MHS2003), pp.89-94, 2003. 